\section{Untersuchungsgut, biologisches}

W. G. Guder

München, Deutschland

Synonym(e) Material, biologisches

Englischer Begriff biological specimen; medical specimen

Definition $\triangleright$ Probe $(\triangleright$ Spezimen), die aus lebenden oder toten Lebewesen stammen und für eine Untersuchung bestimmt sind. Je nach Untersuchungsart wird zwischen medizinischem oder biologischem Untersuchungsgut unterschieden.
Beschreibung Gemäß einer Verordnung zum Versand von Untersuchungsmaterial müssen die Verpackungen mit einem Zeichen für biologisches (oder medizinisches) Untersuchungsgut markiert werden. Dies umfasst alle Arten von Proben, flüssig oder fest, die zum Zweck der Untersuchung versandt werden.

\section{Literatur}

Deutsche Post AG (1996) Regelungen über den Postversand von medizinischem und biologischem Untersuchungsgut. Mitteilungsbl Dtsch Post AG 34:545

EN 829 (1996) In vitro Diagnostic Systems. Transport Packages for Medical and Biological Specimens. Requirements, tests. European Commitee for Standardization (CEN), Brüssel 